\title{
Optics
}

2014; 3(6-1): 2-9

Published online July 30, 2014 (http://www.sciencepublishinggroup.com/j/optics)

doi: 10.11648/j.optics.s.2014030601.12

ISSN: 2328-7780 (Print); ISSN: 2328-7810 (Online)

\section{Exciton states spectroscopy in quasi - zero - dimensional nanostructures: Theory}

\author{
Sergey I. Pokutnyi \\ Chuiko Institute of Surface Chemistry, National Academy of Sciences of Ukraine, 17 General Naumov Str., UA - 03164 Kyiv, Ukraine
}

Email address:

Pokutnyi_Sergey@inbox.ru

\section{To cite this article:}

Sergey I. Pokutnyi. Exciton States Spectroscopy in Quasi - Zero - Dimensional Nanostructures: Theory. Optics. Special Issue: Optics and Spectroscopy of the Charge Carriers and Excitons States in Quasi - Zero - Dimensional Nanostructures. Vol. 3, No. 6-1, 2014 , pp. 2-9. doi: 10.11648/j.optics.s.2014030601.12

\begin{abstract}
The theory of exciton states in a quantum dot under conditions of dominating polarization interaction of an electron and a hole with a spherical (quantum dot - dielectric matrix) interface is developed. An shown, that the energy spectrum of heavy hole in the valence band quantum dot is equivalent to the spectrum of hole carrying out oscillator vibrations in the adiabatic electron potential. In the framework of the dipole approximation studied interband absorption of light in a quasi - zero - dimensional nanosystems. We show that the absorption and emission edge of quantum dots is formed by two transitions of comparable intensity from different hole size - quantization levels and into a lower electron size quantization level. Propose a theoretical prospect of using hole transitions between equidistant series of quantum levels observed in nanocrystals for desining a nanolaser.
\end{abstract}

Keywords: Excitons, Energy Spectrum, Exciton Binding Energy, Absorption of Light, Nanolaser

\section{Introduction}

The optical properties of quasi - zero - dimensional structures consisting of spherical semiconductor nanocrystals (SNs) (quantum dots (QDs)) and dielectric nanocrystals of radius $a \sim 1-10 \mathrm{~nm}$, grown in transparent dielectric matrices, are currently being intensively studied [1-12]. The reason for these studies is that heterophase systems are promising materials for creating new nonlinear optoelectronic elements, in particular elements for controlling optical signals in optical lasers or optical computers [1-18].

Since the energy gap of a semiconductor is much smaller than in the dielectric host, the motion of carriers in the semiconductor microcrystal is restricted by its volume. Optical and nonlinear optical properties of heterophase systems are determined by the energy spectrum of spatially limited electron - hole pairs (EHP) and excitons) Refs. [1-22]. Quantum well effects have been found by optical spectroscopic studies in the energy spectrum of electrons and excitons [1-6] in such quasi - zero - dimensional structures.

The energy spectrum of an exciton in a small $\mathrm{SN}$ was obtained in Refs. [4-22] as a function of the SN radius $a$.
The mentioned spectrum has been obtained using the adiabatic approximation, under the assumption that $m_{e} \ll m_{h}$, where $m_{e}$ and $m_{h}$ are the effective masses of an electron and a hole in a SN. In the exciton Hamiltonian, the Coulomb interaction between an electron and hole, as well as the polarization interaction of the electron and the hole with the surface of the $\mathrm{SN}$, have been taken into account. The first order perturbation theory on the electron wave function of a spherical potential well of infinite depth has been used.

In Ref. [22] the exciton energy spectrum of a small SN, under the assumption that the electron could leave the SN volume for the ambient dielectric medium, has been obtained. The problem solved in Ref. [22] was that of the electron energy spectrum in small SN. In these studies the finite of the potential barrier $\left(V_{0}\right)$ at the boundary between the SN and the dielectric matrix, and the associated penetration of the ambient host by the electron, have been taken into account. The electron wave functions were obtained in Refs. [20-22] for an electron travelling both in the $\mathrm{SN}$ and in the ambient dielectric host.

The motion of charge carriers in semiconductor nanocrystals, like in quantum dots, is limited in three directions. Therefore the energy spectrum of charge carriers is discrete in $\mathrm{SNs}$ with linear dimension of the 
order of 1-10 $\mathrm{nm}$. In our opinion this property may be used for designing optical nanolasers or optical computers [1, 13]. In this context we point out that the size (i.e., radius) $a$ of a quantum dot should be in the range of a few nanometers in order to provide that the difference, i.e., energy splitting $\Delta E_{e(h)}$, between electron and hole levels are of the order of a few $k_{B} T_{0}$ at the room temperature $T_{0}\left(k_{B}\right.$ is the Boltsman constant).

In experiments reported in Ref. [2], an interesting energetic structure was found which consisted of an equidistant series of quantum levels. The above structure is caused by quantization of the heavy hole energy spectrum in the adiabatic potential of the electron. In [13] presents the theoretical prospect of using semiconductor nanocrystals for designing nanolasers.

The theory of exciton states in a QD under conditions of dominating polarization interaction of an electron and a hole with a spherical (quantum dot - dielectric matrix) interface is developed [10-27]. In [28], a new modified effective mass method was proposed to describe the exciton energy spectrum in semiconductor QDs with radii of $a \approx a_{e x}$ ( $a_{e x}$ is the exciton Bohr radius in the semiconductor material contained in the QD volume). It was shown that, within a model in which the QD is represented as an infinitely deep potential well, the effective mass approximation can be applied to the description of an exciton in QDs with radii $a$ comparable to the exciton Bohr radius $a_{\mathrm{ex}}$, assuming that the reduced effective exciton mass is a function of the radius $a, \mu=\mu(a)$ [28]. Contributions of a kinetic energy of an electron, energy of a Coulomb interaction between an electron and a hole, and energy of their polarization interaction with a QD surface to an energy spectrum of an exciton in a QD are analyzed [26-29].

In the adiabatic approximation and within the modified effective mass method [28], an expression for the binding energy of an exciton whose electron and hole move in the semiconductor QD volume was derived in [29]. In [29], the effect of significantly increasing the exciton-binding energy in cadmium selenide and sulfide QDs with radii $a$, comparable to the exciton Bohr radii aex, relative to the exciton- binding energy in cadmium selenide and sulfide single crystals (by factors of 7.4 and 4.5 , respectively) was also detected.

A theory of the size quantization Stark effect in SNs has been developed for the case of dominating polarization interaction of an electron and a hole with the nanocrystal surface [17]. A shift of electron and hole quantum well levels in a $\mathrm{SN}$ in the interband absorption range in a uniform external electric field is determined by the quantum-confinement quadratic Stark effect. An electrooptical method is proposed, making it possible to estimate the characteristic quantum dot radius at which three-dimensional excitons can exist [17]. Electrooptical properties of quasi-zero-dimensional nanosystems containing SNs are governed by the quantum-confinement quadratic Stark effect in the range of interband absorption [17].
The theory of an exciton formed from spatially separated electron and hole (the hole is in the quantum dot volume, and the electron is localized at the outer spherical quantum dot-dielectric matrix interface) is developed within the modified effective mass method. The effect of significantly increasing the exciton binding energy in quantum dots of zinc selenide, synthesized in a borosilicate glass matrix, relative to that in a zinc selenide single crystal is revealed. It was shown that the short-wavelength shift of the peak of the low-temperature luminescence spectrum of samples containing zinc-selenide quantum dots, observed under the experimental conditions, is caused by quantum confinement of the ground-state energy of the exciton with a spatially separated electron and hole [23,24].

The paper is organized as follows. In Section 2 we discuss the energy spectrum of exciton states. In section 3 we consider interband absorption and emission of light in SNs. The concept of using CdS nanocrystals as active lasing medium for constructing nanolasers is presented in Section 4. The theory of an exciton and biexciton formed from spatially separated electrons and holes is developed within the modified effective mass method in Section 5.

\section{Spectrum of an Exciton in a Quasi - Zero - Dimensional Nanosystem}

A simple model of a quasi - zero - dimensional structure in the form of a neutral spherical $\mathrm{SN}$ of radius $a$ and permittivity $\varepsilon_{2}$, embedded in a medium with permittivity $\varepsilon_{1}$, was discussed in Refs. [7-12]. An electron (e) and a hole $(h)$ with effective masses $m_{e}$ and $m_{h}$ were assumed to travel within the $\mathrm{SN}$; we use $r_{e}$ and $r_{h}$ to denote the distances of an electron and a hole from the center of the $\mathrm{SN}$, respectively (see Fig. 1). We assume that the permittivities satisfy the relation $\varepsilon_{2} \gg \varepsilon_{1}$, and that the conduction and valence bands are parabolic. In this model, in the framework of the effective mass approximation, the exciton ( EHP) Hamiltonian takes the form [19-22]

$$
\begin{aligned}
H=-\frac{\hbar^{2}}{2 m_{e}} \nabla_{e}^{2}- & \frac{\hbar^{2}}{2 m_{h}} \nabla_{h}^{2}+E_{g}+V_{e h}\left(r_{e}, r_{h}\right)+V_{h \hat{h}}\left(r_{h}, a\right) \\
& +V_{e \dot{e}}\left(r_{e}, a\right)+ \\
& +V_{e \hat{h}}\left(r_{e}, r_{h}, a\right)+V_{h e}\left(r_{e}, r_{h} a\right)
\end{aligned}
$$

where the first two terms represent the kinetic energy of the electron and the hole, $V_{e h}\left(r_{e}, r_{h}\right)$ is the energy of the Coulomb interaction between the hole and the electron

$$
V_{e h}\left(r_{e}, r_{h}\right)=-\frac{e^{2}}{\varepsilon_{2} a} \frac{a}{\left(r_{e}^{2}-2 r_{e} r_{h} \cos \theta+r_{h}^{2}\right)^{1 / 2}}
$$

where the angle $\theta$ between the vector $\left(r_{e}\right)$ and $\left(r_{h}\right)$ and $V_{h \bar{h}}\left(r_{h}, a\right)$ are the energies of interaction of the electron and the hole with their own images, $V_{e h}\left(, r_{e}, r_{h}, a\right)$ and $V_{h e}\left(r_{e}, r_{h}, a\right)$ are the energies of interactions with "strange" images, and $E_{g}$ is the bandgap in the infinite semiconductor with permittivity $\varepsilon_{2}$. (see Fig. 1). 


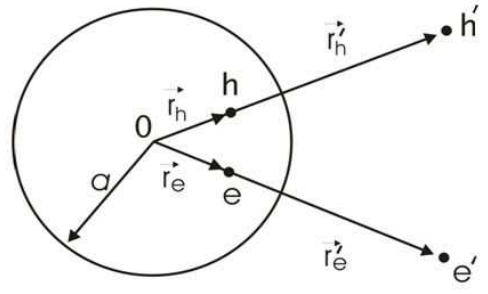

Fig. 1. Schematic diagram of an exciton in a spherical semiconductor nanocrystal. The radius vectors $r_{e}$ and $r_{h}$ determine the distance of the electron $e$ and the hole $h$ from the center of a nanocrystal of radius $a$. The image charges $e^{\prime}=\left(a / r_{e}\right) e$ and $h^{\prime}=-\left(a / r_{h}\right) e$ are located at the distances $r_{e}^{\prime}=\left(a^{2} / r_{e}\right)$ and $r_{h}^{\prime}=\left(a^{2} / r_{h}\right)$ from the center of the nanocrystal 0 and represent point image charges of the electron and hole, respectively.

For arbitrary $\varepsilon_{1}$ and $\varepsilon_{2}$, the terms in Eq. (1) that describe the energy of the polarization interaction of the electron and hole with the SN surface can be written in an analytic form [19-22] which is particularly simple for the case $\varepsilon_{2} \gg \varepsilon_{1}$ :

$$
\begin{gathered}
V_{h h^{\prime}}\left(r_{h}, a\right)=\frac{e^{2}}{2 \varepsilon_{2} a}\left(\frac{a^{2}}{a^{2}-r_{h}^{2}}+\frac{\varepsilon_{2}}{\varepsilon_{1}}\right), \\
V_{e \dot{e}}\left(r_{e}, a\right)=\frac{e^{2}}{2 \varepsilon_{2} a}\left(\frac{a^{2}}{a^{2}-r_{e}^{2}}+\frac{\varepsilon_{2}}{\varepsilon_{1}}\right), \\
V_{e \hat{h}}=V_{h e ́}=-\frac{e^{2}}{2 \varepsilon_{2} a} \frac{a}{\left[\left(r_{e} r_{h} / a\right)^{2}-2 r_{e} r_{h} \cos \theta+a^{2}\right]^{1 / 2}} .
\end{gathered}
$$

The spectrum of an exciton (EHP) in a small semiconductor microcrystal was investigated in Refs. [19-22] in the case where its size was restricted by the condition

$$
a_{0} \ll a_{h} \ll a \leq a_{e} \cong a_{e x},
$$

where $\quad a_{h}=\varepsilon_{2} \hbar^{2} /\left(m_{h} e^{2}\right), a_{e}=\varepsilon_{2} \hbar^{2} /\left(m_{e} e^{2}\right) \quad$ and $a_{e x}=\varepsilon_{2} \hbar^{2} /\left(\mu e^{2}\right)$ are the Bohr radii of the hole, electron and exciton in the semiconductor with permittivity $\varepsilon_{2}$, respectively; $e$ is the electron charge, $\mu=m_{e} m_{h} /\left(m_{e}+m_{h}\right)$ is the reduced effective mass of the EHP, and $a_{0}$ is a characteristic size that is of the order of the interatomic distance. When the condition (6) is satisfied, the polarization interaction is a significant part in the potential energy of the Hamiltonian (1). The above inequalities also enable us to examine the motion of an electron and a hole in the effective mass approximation. The validity of Eq. (6) further enables us to consider the motion of a heavy hole $\left(m_{h} \gg m_{e}\right)$ in the electron potential averaged over the motion of the electron (adiabatic approximation).

The adiabatic approximation can be used if we assume that the electron kinetic energy is the largest term and take the last four terms in Eq. (1) together with nonadiabatic operator as a perturbation. Then, taking into account only the first order of the perturbation theory, one can easily obtain the spectrum of an exciton (EHP), $E_{n_{e}, l_{e}, m_{e}}^{n_{h} l_{h}, m_{h}}(a)$, in the state $\left(n_{e}, l_{e}, m_{e} ; n_{h}, l_{h}, m_{h}\right)$ (here $n_{e}, l_{e}, m_{e}$ and $n_{h}, l_{h}, m_{h}$ are the main, orbital and magnetic quantum numbers of an electron and a hole) [19-22, 26-29]:

$$
\begin{aligned}
& E_{n_{e}, l_{e}, m_{e}}^{n_{h}, l_{h}, m_{h}}(a)=\frac{\hbar^{2}}{2 m_{e} a^{2}} u_{n_{e}, l_{e}}^{2}+ \\
& \bar{V}_{e \dot{e}}(a)+\lambda_{n_{e}, l_{e}, m_{e}}^{n_{h}, l_{h}, m_{h}}(a)+E_{g},
\end{aligned}
$$

where the first term is the kinetic energy of an electron in an infinite spherical well, and $\bar{V}_{e e}(a)$ is the average value of the electron interaction with the self - image with the infinitely deep spherical well functions

$$
\Psi_{n_{e}}, l_{e}, m_{e}=\frac{\sqrt{2 / r_{e}}}{a} Y_{l_{e}, m_{e}}(\theta, u) \frac{J_{l_{e+1 / 2}}\left(u_{n_{e}, l_{e}} r_{e} / a\right)}{J_{l_{e}+3 / 2}\left(u_{n_{e}, l_{e}}\right)},
$$

where $Y_{l_{e}, m_{e}}$ are normalized spherical functions, $u_{n_{e}, l_{e}}$ are roots of the Bessel function $J_{l_{e}+1 / 2}\left(u_{n_{e}, l_{e}}\right)=0$. The quantity $\lambda_{n_{e}, l_{e}, m_{e}}^{n_{h}, l_{h}, m_{h}}(a)$ is an eigenvalue of the heavy hole Hamiltonian

$$
H_{h}=-\frac{\hbar^{2}}{2 m_{h}} \nabla_{h}^{2}+V_{h \hat{h}}\left(r_{h}, a\right)+\bar{V}_{n_{e}, l_{e}, m_{e}}\left(r_{h}, a\right),
$$

where

$\bar{V}_{n_{e}, l_{e}, m_{e}}\left(r_{h}, a\right)=\bar{V}_{e h}\left(r_{h}, a\right)+\bar{V}_{e \hat{h}}\left(r_{h}, a\right)+\bar{V}_{h e ́}\left(r_{h}, a\right)$

is the average value of the Coulomb interaction and of the electron and hole interaction with "alien" images on "free" electron states (8).

Quantitative results for the EHP spectrum (7) are obtained here only for the simple case $l_{e}=0$. Using expressions (3) - (5), (8) and (10) one can obtain

$$
\begin{gathered}
\bar{V}_{e h}^{n_{e}, 0,0}(x, s)=s^{-1}\left[\frac{\sin \left(2 \pi n_{e} x\right)}{\pi n_{e} x}-2 \operatorname{Ci}\left(2 \pi n_{e} x\right)+\right. \\
\left.2 \operatorname{Ci}\left(2 \pi n_{e}\right)+2 \ln x-2\right], \\
\bar{V}_{e \bar{h}}^{n_{e}, 0,0}(x, s)+\bar{V}_{h e}^{n_{e}, 0,0}(x, S)=-2 s^{-1}, \\
\bar{V}_{e \dot{e}}^{n_{e}, 0,0}(x, s)=s^{-1}\left(Z_{n_{e}, 0}+\frac{\varepsilon_{2}}{\varepsilon_{1}}\right), \\
\bar{V}_{h \bar{h}}(x, s)=s^{-1}\left(\frac{1}{1-x^{2}}+\frac{\varepsilon_{2}}{\varepsilon_{1}}\right)
\end{gathered}
$$

where

$$
Z_{n_{e}, 0}=2 \int_{0}^{1} d x_{e} \frac{\sin ^{2}\left(\pi n_{e} x_{e}\right)}{1-x_{e}^{2}}
$$

and $\mathrm{Ci}(\mathrm{y})$ is the integral cosine. Here and below the energy is measured in units $R y_{h}=\hbar^{2} / 2 m_{h} a_{h}^{2}$ and nondimensional values of the length $x=\left(r_{h} / a\right)$ and $s=\left(a / a_{h}\right)$ are used.

Note that in the approximation considered the interaction of an electron with the images (its own and "alien") (12), (13) and the interaction of a hole with an "alien" image (12) yield a constant addition $\sim S^{-1}$ to the hole energy. 
Taking into account formulae (3) and (10) - (12), we write the potential energy in the heavy hole Hamiltonian (9) as follows

$$
\begin{gathered}
\bar{U}^{n_{e, 0,0}}(x, s)=V_{h \dot{h}}(x, s)+\bar{V}^{n_{e}, 0,0}(x, s)= \\
=s^{-1}\left[\frac{1}{1-x^{2}}+\frac{\sin \left(2 \pi n_{e} x\right)}{\pi n_{e} x}-2 C i\left(2 \pi n_{e} x\right)+2 \operatorname{Ci}\left(2 \pi n_{e}\right)+\right. \\
\left.2 \ln x+\frac{\varepsilon_{2}}{\varepsilon_{1}}-4\right] .
\end{gathered}
$$

The minimum of the potential energy (14)

$$
\bar{U}_{\min }^{n_{e}, 0,0}(s)=\bar{U}^{n_{e}, 0,0}(x=0, s)=\frac{P_{n_{e}, 0}}{s},
$$

Where

$$
P_{n_{e}, 0}=2 C i\left(2 \pi n_{e}\right)-2 \ln \left(2 \pi n_{e}\right)-2 \gamma+\frac{\varepsilon_{2}}{\varepsilon_{1}}-1,
$$

( $\gamma=0,577$ is the Euler constant) is reached at the point $x$ $=0$. A series expansion of the potential (14) with respect to the parameter $x^{2} \ll 1$ with accurancy up to the first two terms gives the hole spectrum $\lambda_{n_{e}, 0,0}^{t_{h}}(\bar{s})$ in oscillator form [19-22]:

$$
\lambda_{n_{e}, 0,0}^{t_{h}}(\bar{s})=\frac{P_{n_{e}, 0}}{\bar{s}}+\bar{\omega}\left(\bar{s}, n_{e}\right)\left(t_{h}+\frac{3}{2}\right),
$$

where

$$
\bar{\omega}\left(\bar{s}, n_{e}\right)=2.232\left(1+\frac{2}{3} \pi^{2} n_{e}^{2}\right)^{1 / 2} \bar{s}^{-3 / 2},
$$

and $\bar{\omega}\left(\bar{s}, n_{e}\right)$ is the frequency of the hole oscillator vibrations, $t_{h}=2_{n_{r}}+l_{h}=0,1, \ldots$ is the hole main quantum number $\left(n_{r}\right.$ is the hole radial quantum number).

Taking into account formulae (13), (15) and (16) we obtain the EHP spectrum $\left[E_{n_{e}, l_{e}=0}^{t_{h}}(\bar{s})\right]$ in the state $\left(n_{e}, l_{e}=0 ; t_{h}\right)$ for the $\mathrm{SN}$ of $\operatorname{radius}\left(\bar{s}=\bar{a} / a_{h}\right)$ [19-22]:

$$
\begin{aligned}
E_{n_{e}, l_{e}=0}^{t_{h}}(\bar{s})= & E_{g}+\frac{\pi^{2} n_{e}^{2}}{\bar{s}^{2}} K \beta+\bar{s}^{-1}\left(Z_{n_{e}, 0}+P_{n_{e}, 0}+\frac{\varepsilon_{2}}{\varepsilon_{1}}\right)+ \\
& \bar{\omega}\left(\bar{s}, n_{e}\right)\left(t_{h}+\frac{3}{2}\right),
\end{aligned}
$$

where $\mathrm{K}=0.67$ represents the spread of the $\mathrm{SN}$ radii [4] and $\beta=m_{e} / m_{h}$. Moreover, numerical analysis of $\mathrm{x}-$ ray data [2], which took the spread of the $\mathrm{SN}$ radii into account, shows that the mean radius evaluated over the Lifshits Slezov distribution was $\bar{s}=0,86 s$ [2], where s is the SN radius obtained in the small variance approximation.

The main contribution to the EHP spectrum (17) is provided by second term (electron kinetic energy), which comes from the purely spatial restrictions imposed on the quantization region. The last two terms, which are associated with the Coulomb and polarization interactions between the electron and the hole, are regarded as corrections. The polarization interaction between hole and electron, on the one hand, and the surface of the SN, on the other, provides, like the size quantization of carriers, a contribution to the renormalization of the energy gap of the $\mathrm{SN}$ given by Eq. (17). The polarization interaction, which is of the same order of magnitude as the exciton binding energy in the $\mathrm{SN}$, in this case is found to be much smaller than the size quantization energy of the charge carriers in the SN.

We also note that the exciton spectrum (17) is valid only for low - lying states of the exciton $\left(n_{e}, 0 ; t_{h}\right)$ in the $\mathrm{SN}$, for which $\left(E_{n_{e}, 0}-E_{g}\right) \ll V_{0}$, where $V_{0}$ is the depth of the potential well for an electron in the $\mathrm{SN}$ (e.g., in the cadmium sulphide $\mathrm{SN}, V_{0}=2.3-2.5 \mathrm{eV}$ when the condition (6) is satisfied [30]).

In Refs. [2] interband absorption spectra of CdS SNs $\left(\varepsilon_{2}=9.3\right)$ of sizes varying from 1 to $10^{2} \mathrm{~nm}$ dispersed in a transparent dielectric matrix of silicate glass were investigated. The effective masses of electrons and holes in $\mathrm{CdS}$ were, respectively, $m_{e} / m_{0}=0.205$ and $m_{h} / m_{0}=5$ (i.e., $m_{e} \ll m_{h}$ ). The dimensional quantization phenomenon found in ref. [2] for the electron energy spectrum was qualitatively described by the formula

$$
E_{n_{e}, l_{e}}(a)=E_{g}+\frac{\hbar^{2}}{2 \mu a^{2}} u_{n_{e}, l_{e}}^{2}
$$

In this case in the region of SN sizes $a$ comparable with the exciton radius, $a \leq a_{e x} \cong 3 \mathrm{~nm}$, a deviation of the experimental points from the theoretical dependence (18) was observed. The spectrum of electron - hole pairs in the interval of SN sizes $a \leq a_{e x}$ calculated in Refs. [19-22] gave a qualitatively correct description of the dependence of the experimental spectrum [2] on the SN radius $a$.

In the experiment reported in Ref. [2], an energy structure consisting of an equidistant series of levels was found in the region if transitions to the lower level of dimensional quantization of the electron $\left(n_{e}=1\right)$. The above structure is caused by quantization of the hole energy spectrum in the adiabatic potential of the electron.

From the comparison of formula (16) (for $n_{e}=1$ ) with the experimental dependence of the splitting magnitude on the SN size $\bar{a}$ obtained in Ref. [5], it follows that for SNs of radii $1.5 \leq \bar{a} \leq 3.0 \mathrm{~nm}$, the splitting $\bar{\omega}(\bar{s})$ (Eq. (16)) is in good agreement with the experimental data [2] and differs from the latter only slightly $(\leq 4 \%)$.

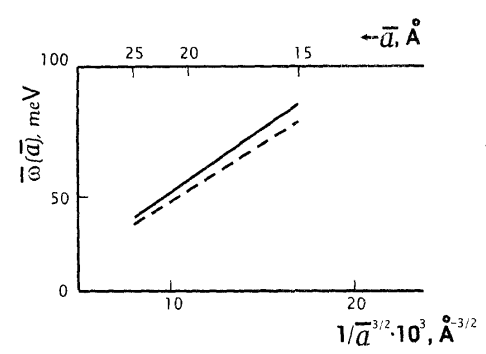

Fig. 2. The dependence of the splitting $\bar{\omega}(\bar{a})$ (16) of the radius of the nanocrystal $\bar{a}$. The solid line shows the experimental dependence $\bar{\omega}(\bar{a})[2]$

\section{Interband Absorption of Light in Nanocrystal}

The interband absorption of light in SNs was studied theoretically in [14-16] using the dipole approximation in 
the framework of the model considered here, and under the assumption that the absorption length $\lambda \gg a$.

An expression for the quantity $\mathrm{K}(\bar{s}, \omega)$ defined by the hole optical transition from the energy level $t_{h}=2 n_{h}$ to the lowest electron level $\left(n_{e}=1, l_{e}=m_{e}=0\right)$ was derived in the following form [15]:

$$
\begin{gathered}
K(\bar{s}, \omega)=A \sum_{n_{h}} L_{n_{h}}(\bar{S}) \delta\left[\Delta(\omega)-\frac{\pi^{2}}{\bar{s}^{2}} \frac{m_{h}}{m_{e}}-\frac{1}{\bar{s}}\left(Z_{1,0}+P_{1,0}+\right.\right. \\
\left.\left.\frac{\varepsilon_{2}}{\varepsilon_{1}}\right)-\bar{\omega}\left(\bar{s}, n_{e}=1\right)\left(2 n_{h}+3 / 2\right)\right]
\end{gathered}
$$

where $\Delta(\omega)=\hbar \omega-E_{g}, \omega$ - incident light frequency, and $A$ is proportional to the square of the absolute value of the dipole moment matrix element calculated with Bloch functions. The quantity $K(\bar{s}, \omega)(19)$ connects the energy absorbed by $\mathrm{SN}$ in a time unit with the time average of electric field square of incident wave. Moreover, the product of $K(\bar{s}, \omega)$ and SN concentration in the dielectric matrix gives electric conductivity of the considered quasi zero - dimensional system for the frequency $\omega$, which is connected with light absorption coefficient in the usual way.

We determine the quantity $K(\bar{s}, \omega)$ (19) corresponding to hole optical transition from the energy level $t_{h}=2 n_{h}$ to the lowest electron level $\left(n_{e}=1, l_{e}=m_{e}=0\right)$. In this case, the expression for the quantity $L_{n_{h}}(\bar{s})$, given by the square of the overlap integral of the electron and hole wave functions, take the form [14-16]

$$
L_{n_{h}}(\bar{S})=\frac{2 \pi^{5 / 2}}{\left(1+\frac{2}{3} \pi^{2}\right)^{3 / 4}} \frac{n_{h}+1}{2^{2} n_{h} n_{h} !}(\bar{S})^{-3 / 4}
$$

In the interband optical absorption spectrum of $\mathrm{SN}$, the radius of which fulfills condition (7), each line corresponding to given values of the radial $n_{e}$ and orbital $l_{e}$ quantum numbers turns into a series of close lying equidistant levels, corresponding to various values of the main hole quantum number $t_{h}$. This conclusion follows from (15), (16) and (19), and is a direct consequence of the Coulomb and polarization interactions of an electron and a hole in $\mathrm{SN}$.

We can estimate value of the overlap integral square $(K(\bar{s}, \omega) / A)$ using (19), (20) and the experimental data taken from Ref. [2]. For the hole transitions from the equidistant quantum levers: $\quad\left(n_{h}=0 ; l_{h}=m_{h}=\right.$ $0),\left(n_{h}=1 ; l_{h}=m_{h}=0\right)$,

$\left(n_{h}=2 ; l_{h}=m_{h}=0\right),\left(n_{h}=3 ; l_{h}=m_{h}=0\right)$, to the lowest electron size -quantized level $\left(n_{e}=1, l_{e}=m_{e}=\right.$ $0)$, we have

$$
\frac{K(\bar{s}, \omega)}{A}=L_{1}(\bar{s})+L_{2}(\bar{s})+L_{3}(\bar{s}),
$$

where $L_{0}=7.66 \bar{s}^{-3 / 4}, L_{1}=0.5 L_{0}, L_{2}=9.4 \cdot 10^{-2} L_{0}$, $L_{3}=10^{-2} L_{0}$. From above expression, it follows that the main contribution in the light absorption coefficient is a cadmium sulphide SN with the radius $s$ (6) comes from the hole spectral lines corresponding to quantum numbers $\left(n_{h}=0 ; l_{h}=m_{h}=0\right)$ and $\left(n_{h}=1 ; l_{h}=m_{h}=0\right)$, the transition oscillator strengths of which are dominant. The contribution of higher exited hole lines $\left(n_{h} \geq 2 ; l_{h}=\right.$ $\left.m_{h}=0\right)$ is negligible.

This way, in the framework of the considered model of the quasi - one - dimensional system it was shown that the absorption and emission edge of a cadmium sulphide SN is formed by two transitions of comparable intensities.

\section{Nanolaser on Heavy Hole Transition in Semiconductor Nanocrystals}

We assume that CdS microcrystals have a direct band structure, like bulk CdS. The extrema of the conduction and valence bands are placed in the centre of the Brillouin zone. Moreover, CdS is wide - gap semiconductor ( $E_{g}=$ $2.58 \mathrm{eV}$ ) in which nonparabolicity effect are weak. At the same time, the dispersion laws near the bottom of the conduction band and the top of the valence band can be considered as parabolic [2].

In the construction of the theory, it was assumed that the electron and hole bands are parabolic. The nonparabolicity parameter $j$ of such bands for EHP energy $E_{1,0}^{t_{h}}(a)$ (17) in the group state, which is obtained from the experiments of Refs. [2] in SN of size $a \sim a_{\text {ex }}$ takes a small value

$$
j(a)=\frac{E_{1,1}^{t_{h}}-E_{g}}{E_{g}} \leq 8 \% .
$$

This relation gives a basis for the assumption that the valence and conduction bands are, to a high degree of accuracy, parabolic.

In Fig.3, the energy diagram of the optical transitions in CdS SN of radius $a$ fulfilling the condition (6) is shown Ref. [13]. The electron energy spectrum $E_{n_{e}, l_{e}}(a)$ (18) in the conduction band of a SN of the radius $a$ comparable to the linear dimension of quasiparticles in semiconductors is determined only by the size quantization effect. The energy spectrum $\lambda_{n_{e}, 0,0}^{t_{h}}$ of heavy hole in the valence band is equivalent to the spectrum of hole carrying out oscillator vibrations with frequency $\bar{\omega}\left(\bar{S}, n_{e}\right)$ (Eq.(16)) in the adiabatic electron potential.

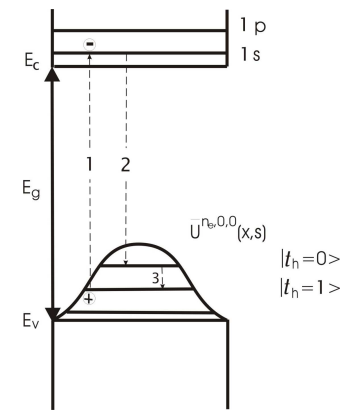

Fig. 3. The scheme of the optical transition of an electron and a hole in a $C d S$ nanocrystal. The electron energy spectrum $E_{n_{e}, l_{e}}(a)$ (18) and the hole potential energy $\bar{U}^{n_{e, 0,0}}(x, s)(14)$ are shown; $E_{g}-$ bandgap width. The electron states in the conduction band are labeled as $(1 s)$ and $(1 \mathrm{p})$. The hole states in the valence band are denoted as $\left|t_{h}=0\right\rangle$ and $\mid t_{h}=$ 1). Transitions are labelled as follows: "1" - pumping, "2"- creating inverse population, " 3 " - lasing 
On the basis of presented results we can formulate an action scheme for a laser designed on CdS quantum dots [13] grown in boric - silicate glass matrices [1-6]:

1. Pumping: the hole transition from energy level $\lambda_{n_{e}, 0,0}^{t_{h}}(a)$ to electron state $E_{n_{e}=1, l_{e}=0}(a)$ (1s state) in conduction band with energy (transition " 1 " in Fig.3):

$$
h v_{1}(\bar{s})=E_{g}+E_{1,0}(\bar{s})+\lambda_{1,0,0}^{1}(\bar{s})
$$

2. Creation of inverse population on the hole level $\left|t_{h}=0\right\rangle$ by means of electron transition from the energy level $E_{1,0}(\bar{S})$, with spin flip [31], to the hole energy level $\lambda_{1,0,0}^{0}(\bar{S})$ (transition " 2 " in Fig.3). The energy of this transition

$$
\hbar v_{2}(\bar{s})=E_{g}+E_{1,0}(\bar{s})+\lambda_{1,0,0}^{0}(\bar{s})
$$

3. Lasing step: transition of the hole in the valence band between equidistant levels $\left|t_{h}=0\right\rangle \rightarrow$ $\left|t_{h}=1\right\rangle$ (transition " 3 " in Fig.3) separated by the energy

$$
\Delta(\bar{s})=\hbar \bar{\omega}\left(n_{e}=1, \bar{s}\right) .
$$

In the experimental conditions from Refs. [1-6] at liquid nitrogen temperatures $(T=77 \mathrm{~K})$, the distance $\Delta E_{e}$ between electron energy levels $\left(n_{e}=1, l_{e}=1\right)(1 \mathrm{p}$ - state) and $\left(n_{e}=1, l_{e}=0\right)$ (1s state), according to Eq. (18)

$$
\Delta E_{e}=\frac{\left(u_{1,1}^{2}+\pi^{2}\right)\left(m_{h} / m_{e}\right) R y_{h}}{\overline{S^{2}}} \cong 480 \mathrm{meV},
$$

where $u_{1,1}=4.493$ [32]. The distance $\Delta(\bar{s})$ between the hole energy levels (23) is $\Delta(\bar{s})=\hbar \bar{\omega}\left(n_{e}=1, \bar{s}\right) \cong$ $53 \mathrm{meV}$.

For $\mathrm{SN}$ of the radius $a=2 \mathrm{~nm}, \Delta(\bar{s})$ is several times greater than the thermal energy $k_{B} T$ at $T=77 \mathrm{~K}$. In a nanolaser on $\mathrm{CdS}$ nanocrystals, the pumping energy $\mathrm{h} v_{1} \cong 3.11 \mathrm{eV}$ (22) would correspond to visible light, and lasing at energy $\Delta(\bar{s}) \cong 53 \mathrm{meV}$ would occur in the infrared range [13].

\section{Excitons and Biexcitons Formed from Spatially Separated Electrons and Holes in Quasi - Zero - Dimensional Nanosystems}

In [28], a new modified effective mass method was proposed to describe the exciton energy spectrum in semiconductor QDs with radii of $a \approx a_{e x}\left(a_{e x}\right.$ is the exciton Bohr radius in the semiconductor material contained in the QD volume). It was shown that, within a model in which the QD is represented as an infinitely deep potential well, the effective mass approximation can be applied to the description of an exciton in QDs with radii $a$ comparable to the exciton Bohr radius $a_{\mathrm{ex}}$, assuming that the reduced effective exciton mass is a function of the radius $a, \mu=$ $\mu(a)$.
In the adiabatic approximation and within the modified effective mass method [28], an expression for the binding energy of an exciton whose electron and hole move in the semiconductor QD volume was derived in [29]. In [29], the effect of significantly increasing the exciton-binding energy in cadmium selenide and sulfide QDs with radii $a$, comparable to the exciton Bohr radii aex, relative to the exciton- binding energy in cadmium selenide and sulfide single crystals (by factors of 7.4 and 4.5 , respectively) was also detected.

In the experimental study [6], it was found that excess electrons produced during interband excitation of the cadmium sulfide QD have a finite probability of overcoming the potential barrier and penetrating into the borosilicate glass matrix into which the QD is immersed. In experimental studies [6] of glass samples with cadmium-sulfide and zinc-selenide QDs, it was found that the electron can be localized in the polarization well near the outer QD surface, while the hole moves within the QD volume.

In [6], the optical properties of borosilicate glass samples containing QD zinc selenide are experimentally studied. The average radii of such QDs are in the range $a \approx 2.0-4.8$ $\mathrm{nm}$. In this case, the values of $a$ are comparable to the exciton Bohr radius $a_{e x} \approx 3.7 \mathrm{~nm}$ in a $\mathrm{ZnSe}$ single crystal. At low QD concentrations, when the optical properties of the samples are mainly controlled by those of individual QDs in the borosilicate glass matrix, a shift of the peak of the low temperature luminescence spectrum to the short wavelength region (with respect to the band gap $E_{g}$ of the zinc selenide - single crystal) was observed. The authors of [6] assumed that this shift is caused by quantum confinement of the energy spectra of the electron and hole localized near the spherical surface of the QD.

The use of semiconductor nanosystems as the active region of nanolasers is prevented by the low binding energy of the QD exciton [6,13]. Therefore, studies directed toward the search for nanostructures in which a significant increase in the binding energy of QD excitons would be observed are of importance.

The theory of an exciton with a spatially separated electron and hole was developed within the modified effective mass method in which the reduced effective exciton mass is a function of the semiconductor QD radius $a[23,24]$. The effect of significantly increasing the binding energy $\left|E_{e x}(a)\right|$ of the exciton ground state in a nanosystem containing zinc-selenide QDs with radii 4,0 $\leq a \leq 29,8 \mathrm{~nm}$ was detected; in comparison with the exciton binding energy in a zinc-selenide single crystal, the increase factor is 4.1-72.6. It was shown that the effect of significantly increasing the binding energy $\left|E_{e x}(a)\right|$ of the exciton ground state in the nanosystem under study is controlled by two factors: (i) a substantial increase in the electron-hole Coulomb interaction energy and an increase in the energy of the interaction of the electron and hole with "foreign" images (the "dielectric enhancement" effect); (ii) spatial confinement of the quantization region by the QD volume; in this case, as the QD radius $a$ increases, starting from $a \geq$ 
$29.8 \mathrm{~nm}$, the exciton becomes two-dimensional with a ground-state energy that exceeds the exciton binding energy in a zinc-selenide single crystal by almost two orders of magnitude [23, 24].

The average zinc- selenide QD radius was determined by comparing the dependence of the exciton ground-state energy on the QD radius, obtained by the variational method within the modified effective mass method, with the experimental peak of the low-temperature luminescence spectrum [6]. It was shown that the short-wavelength shift of the peak of the low-temperature luminescence spectrum of the samples containing zinc-selenide QDs, which was observed under the experimental conditions, is caused by renormalization of the electron-hole Coulomb interaction energy and also the energy of the polarization interaction of the electron and hole with the spherical QD-dielectric matrix interface, related to spatial confinement of the quantization region by the QD volume. In this case, the hole moves in the QD volume, and the electron is localized at the outer spherical QD-dielectric matrix interface [23, 24].

To apply semiconductor nanosystems containing zincselenide QDs as the active region of lasers, it is required that the exciton binding energy $\left|E_{e x}(a)\right|$ (QD radius $4,0 \leq a \leq 29,8 \mathrm{~nm})$ in the nanosystem be on the order of several $k T_{0}$ at room temperature $T_{0}$ ( $k$ is the Boltzmann constant). Nanosystems consisting of zinc- selenide QDs grown in a borosilicate glass matrix can be used as the active region of semiconductor QD lasers. The parameter $\left|E_{e x}(a) / k T_{0}\right|$ take significant values in the range from 3.1 to $56[23,24]$.

We developed the theory of a biexcitons formed from spatially separated electrons and holes (the hole is in QD volume, and the electron is localized at the outer surface of the QD-matrix interface) in a nanosystem that consists of ZnSe QDs synthesized in a glassy matrix [25]. The dependences of the total energy and binding energy of the singlet ground biexciton state in such a system on the spacing between the QD surfaces and the QD radius are derived by the variational method. It is shown that biexciton formation is of the threshold character and possible in nanosystems, in which the spacing between the QD surfaces is larger than a certain critical spacing [25]. It is shown that the major contribution to the biexciton binding energy is made by the energy of the exchange interaction of electrons with holes and this contribution is much more substantial that the contribution of the energy of Coulomb interaction between the electrons and holes. It is established that the spectral shift of the low-temperature luminescence peak in such QDs is due to quantum confinement of the energy of the biexciton ground state [25].

\section{Conclusion}

The review provides the results of theoretical investigations of excitons states (electron - hole pairs states) in a quasi - zero - dimensional nanosystems consisting of spherical semiconductor nanocrystals (quantum dots) placed in transparent dielectric matrices. The theory of exciton states in a QDs under conditions of dominating polarization interaction of an electron and a hole with a spherical (quantum dot - dielectric matrix) interface are developed. An shown, that the energy spectrum of heavy hole in the valence band QD is equivalent to the spectrum of hole carrying out oscillator vibrations in the adiabatic electron potential.

In the framework of the dipole approximation studied interband absorption of light in a quasi - zero - dimensional nanosystems. An expression is obtained for the light absorption coefficient under conditions where the polarization interaction of the electron and a hole with the surface of the SNs plays role. In result, an shown on the basis of the given model of quasi - zero - dimensional nanosystems that the absorption and emission edge of SNs is formed by two transitions of comparable intensity from different hole size - quantization levels and into a lower electron size - quantization level. Propose a theoretical prospect of using hole transitions between equidistant series of quantum levels observed in nanocrystals for desining a nanolaser.

The theory of an excitons formed from spatially separated electron and hole is developed within the modified effective mass method. The effect of significantly increasing the exciton binding energy in quantum dots of zinc selenide, synthesized in a borosilicate glass matrix, relative to that in a zinc selenide single crystal is revealed. It was shown that the short-wavelength shift of the peak of the low-temperature luminescence spectrum of the samples containing zinc-selenide QDs, which was observed under the experimental conditions, is caused by renormalization of the electron-hole Coulomb interaction energy and also the energy of the polarization interaction of the electron and hole with the spherical QD-dielectric matrix interface, related to spatial confinement of the quantization region by the QD volume. Nanosystems consisting of zinc- selenide QDs grown in a borosilicate glass matrix can be used as the active region of semiconductor QD lasers.

We developed the theory of a biexcitons formed from spatially separated electrons and holes in a nanosystem that consists of $\mathrm{ZnSe}$ QDs synthesized in a glassy matrix . It is shown that the major contribution to the biexciton binding energy is made by the energy of the exchange interaction of electrons with holes and this contribution is much more substantial that the contribution of the energy of Coulomb interaction between the electrons and holes. It is established that the spectral shift of the peak in such QDs is due to quantum confinement of the energy of the biexciton ground state.

\section{References}

[1] Alferov, J.I., (2002). Progress development in semiconductor nanostructures. Physics Uspekhi. 172, 1068 1074. 
[2] Ekimov, A.I., Onushchenko, A.A., Efros, Al.L., (1986). Zize quantization of excitons in nanocrystals. Sov. Phys. JETP Lett. 43, 292-294.

[3] Efros, A.L., Efros, Al.L., Interband absorption light in semiconductor sphere. (1982). Sov. Phys. Semiconductors. $16,955-962$

[4] Kulchin, Y.N., Scherbakov, A.V., Dzyuba, V.P., Voznesenskiy, S.S., (2008). Nonlinear optical properties of heterogeneous nanocomposites. Quantum Electronics. 38, $154-158$.

[5] Dzyuba,V.P., Kulchin, Y.N., Krasnok, A.E., (2010). Nonlinear refractive index of heterogeneous nanocomposites in weak optical fields. Technical Physics Letters. 36, 1-9.

[6] Bondar, V.N., Brodin, M.S., (2010). Optical properties semiconbductor quantum dots. Semiconductors. 44, 884-890.

[7] Pokutnyi, S.I., Theory of excitons in quasi - zero dimensional semiconductors systems; Academperiodika: Kiev, Ukraine, 2003; pp. 24-35, 82-94.

[8] Pokutnyi, S.I., Spectroscopy of electron and exciton states in low - dimensional systems; Academperiodika: Kiev, Ukraine, 2005; pp. 44-55, 142-152.

[9] Pokutnyi, S.I., Optics of nanosystems; Academperiodika: Kiev, Ukraine, 2007; pp. 54-64.

[10] Pokutnyi, S.I., Shpak, A.P., (2005). Influence of polarizing interfction on spectroscopy of excitons in quasi - zero dimensional semiconductors systems. Progr. Phys. Metal. 6, 105-134.

[11] Pokutnyi, S.I., (2005). Optical of spectroscopy of exciton states in quasi - zero - dimensional semiconductors nanosystems (review). Ukr. J. Phys. Rev. 3, 46- 74.

[12] Pokutnyi, S.I., Shpak, A.P., Uvarov, V.N., (2007). Bulk local states in quasi - zero - dimensional nanosystems. Progr. Phys. Metal. 8, 1-20.

[13] Pokutnyi, S.I., (2005). Optical nanolaser heavy hole transitions in in quasi - zero - dimensional semiconductors nanosystems. Phys. Lett. A. 342, 347-352.

[14] Pokutnyi, S.I., (2006). Absorption of light in quantum dots. Semiconductors. 40, 217-224.

[15] Pokutnyi, S.I., (2011). Interband absorption of light in semiconductor quantum dots. J. Nanoscience Lett. 1, 191-198.

[16] Pokutnyi, S.I., Gorbyk, P.P., (2013). Absorption of light in positron and electron states in quasi - zero - dimensional nanosystems. Optics, 2, 47-50.
[17] Pokutnyi, S.I., (2004). Size quantization Stark effect in semiconductor quantum dots. J. Appl. Phys. 96, 1115-1122.

[18] Pokutnyi, S.I., (1997). Absorption of light in in quasi - zero - dimensional nanosystems. Phys. Solid State. 39, 604- 612.

[19] Efremov, N.A., Pokutny, S.I., (1990). Energy spectrum of exciton in spherical particle. Sov. Phys. Solid. State. 32, 955- 964.

[20] Pokutnyi, S.I., (1992). Size quantization of electron-hole pair in semiconductor quantum dots. Phys. Lett. A. 168, 433-438.

[21] Efremov, N.A., Pokutny, S.I., (1985). Macroscopic local charge states in ultradispersion media. Sov. Phys. Solid. State. 27, 27- 35 .

[22] Pokutnyi, S.I., (1995). Size quantization of exciton in semiconductor quantum dots. Phys. Lett. A. 203, 384-401.

[23] Pokutnyi, S.I., (2013). On an exciton with a spatially separated electron and hole in quasi - zero - dimensional semiconductor nanosystems. Semiconductors. 47, 791-798.

[24] Pokutnyi, S.I., (2013). Binding energy of the exciton of a spatially separated electron and hole in quasi - zero dimensional semiconductor nanosystems .Technical Physics Letters. 39, 233-235.

[25] Pokutnyi, S.I., (2013). Biexcitons formed from spatially separated electrons and holes in quasi - zero - dimensional semiconductor nanosystems. Semiconductors. 47, 1626-1635.

[26] Pokutny, S.I., Shpak, A.P., Uvarov, V.N., Pokutnyi, M.S., (2008). Theory of exciton states in quasi - zero dimensional nanosystems. Metal Phys. Advanc. Technol. 30, 459-478.

[27] Pokutnyi, S.I., (2005). Exciton states in quasi - zero dimensional semiconductor nanosystems. Semiconductors. $39,1066-1074$

[28] Pokutnyi, S.I., (2007). Exciton states in semiconductor quantum dots in the framework of the modified effective mass method. Semiconductors. 41, 1323-1331.

[29] Pokutnyi, S.I., (2012). Exciton states in semiconductor quantum dots. Semiconductors. 46, 174-184.

[30] Grabovskis, V., Dzenis, Y., Ekimov, A., (1989). Nonlinear optics of semiconductor - doped glasses . Sov. Phys. Solid State, 31, 272- 275.

[31] Bogdankevich, O., Darznek, S., Eliseev, P., Popuprovodnikovye lazery; Nauka: Moskva, Russia, 1976; pp. 94-98.

[32] Flugge, S., Practical Quantum Machanics I, Springer Verlag: Berlin, Germany, 1971; pp. 111-114. 